\title{
Interrelation of the concept of "uncertainty" in the information theory and theory of measurements
}

\author{
Vadim Kopp ${ }^{1}$, Alexey Balakin ${ }^{1, *}$, Natalya Balakina ${ }^{1}$, and Vladimir Preys ${ }^{2}$ \\ 1"Sevastopol State University", Sevastopol, Russian Federation \\ 2"Tula State University", Tula, Russian Federation
}

\begin{abstract}
The problem of determining the optimal number of multiple measurements based on the type of the error distribution density of the measuring means is considered. The laws of distribution of a random variable are obtained, they ensure the extreme values of dispersion at specified entropy. It is indicated that the given law corresponds to the known compositional one which ensures maximum entropy under the restrictions on variation limits of the random variable and at specified dispersion. In particular, under certain conditions, it corresponds to an abridged normal, uniform and bimodal laws. It is shown that two dispersion values correspond to a single entropy value. This effect is provided due to the fact that the random variable is concentrated on a finite interval. The theorem on the interrelation of entropy and dispersion of a random variable is proved which allows us to reconcile the concept of uncertainty used in the information theory with the same concept used in the up-to-date standards of technical measurements. It is shown that an abridged normal distribution law providing maximum of entropy at specified dispersion, at the same time provides the minimum of dispersion at specified entropy, and the bimodal law provides the maximum of entropy at the maximum of dispersion. The conclusions are based on the solution of two variational problems with isoperimetric constraints. The results of modeling allowing to evaluate the correctness of the conclusions are presented.
\end{abstract}

The issues of quality improvement of products in the machine-tool industry are inextricably linked to the increase of the measurement accuracy $[1,2]$.

In many cases, the toughening of the requirements to the product quality lies in the implementation of more stringent requirements to the tolerances on controlled parameters which in its turn leads to an increase in the requirements for the accuracy of parameters' and product characteristics' measurement. Thus, one of the main tasks facing the metrological services of enterprises is to ensure the required accuracy of measurements performed at various stages of production. Besides, in conditions of acute market competition the requirements imposed on the quality of manufactured products also increase, which in its turn leads to the need of measurements accuracy improvement [3].

Having analyzed the literature we can conclude that improving the accuracy of measurements contributes to the development of both individual branches of the national economy and of thre world scientific and technological progress as a whole.

The methods used today in order to improve the accuracy of measurements are given in the interstate standardization recommendations CIS 64-2003 "SSEUM. Efficiency ensuring in the management of technological processes. Means and methods for measurements accuracy increase" [4].
One of the methods for increasing the accuracy of measurements is to use multiple measurements. This method allows to increase the accuracy of measurement without acquisition of new more accurate means of measurement. However, in this case, the question of the need to determine the minimum number of measurements necessary to ensure a given accuracy arises. For this purpose as indicated in $[5,6]$ it was proposed to determine the number of measurements based on the error distribution density of the measuring instrument on the basis of the expression:

$$
n=\frac{\sigma^{2}}{\varepsilon^{2}},
$$

where $\varepsilon^{2}$ - are the required fidelity values determined from the distribution density and the confidence level, $\sigma^{2}-$ is the dispersion of the measuring instrument.

Thus, the aim of the research is to determine the form of the error distribution density of the measuring instrument and to derive on its basis the relation connecting the dispersion with the differential entropy.

In most cases it is assumed that the law of error distribution of the instrument is normal. In some cases this is permissible and is widely used, and in some cases this introduces significant errors. Indeed, the error of the

\footnotetext{
* Corresponding author: AIBalakin@sevsu.ru
} 
device can not change from minus infinity to plus infinity. We are to consider the fact that the limits of the change in the random variable which is the error of the device are specified in the certification. In addition, it is proposed to determine the distribution density from the maximum entropy [7, 8, 9] of the random variable (for the worst case) by using the principle of Jain's maximum entropy [10]. The density of the distribution is defined as the solution of the variational problem [5]: to determine the density $p(x)$ that delivers the extremum to the functional

$$
\max _{p(x)} \rightarrow\left\{H_{\partial u \phi}(x)=-\int_{a}^{b} p(x) \cdot \log p(x) d x\right\}
$$

under the following restrictions:

$$
\begin{aligned}
& \int_{a}^{b} p(x) d x=1 ; \\
& \int_{a}^{b} x^{2} \cdot p(x) d x=\varepsilon^{2} .
\end{aligned}
$$

The solution of the problem has the form:

$$
p(x)=e^{\mu_{1}-1} \cdot e^{\mu_{2} \cdot x^{2}}, a \leq x \leq b,
$$

where $\mu_{1}, \mu_{2}$ - are the Lagrange multipliers. They are determined from conditions (1), (2).

The solution of the problem shows that the maximum of entropy is achieved with the known compositional distribution law [5], in accordance with which at $\sigma^{2}>\frac{(b-a)^{2}}{12}$ it is two-module, at $\sigma^{2}=\frac{(b-a)^{2}}{12}-$ uniform, at $\sigma^{2}<\frac{(b-a)^{2}}{12}-$ abridged normal. These cases are determined by the sign and the value of the coefficient $\lambda_{2}$. Here $a$ and $b$ are the ranges of variation of the random variable.

As is known, the maximum possible value of entropy $H_{\partial \max }$ is attained at $\sigma^{2}=\frac{(b-a)^{2}}{12}$ and equals to $H_{\partial \max }=\log (b-a)$.

Experimental studies were carried out on two types of measuring instruments: with an inductive converter and with a raster photoelectric converter that confirmed that the law of distribution of their errors is abridged normal [11]. This case is provided when $\mu_{2}<0$.

In itself, the use of entropy as a measure of uncertainty is not entirely convenient and illustrative. As a measure of uncertainty the standard deviation $\sigma$ of the measurement result $[12,13,14]$ is used more often, uniquely related to the dispersion. Then the question arises: how do the entropy and dispersion of a random variable concentrated on a finite interval, and in this case distributed according to the compositional law, correlate.
In determining the entropy natural logarithms were used. As further studies showed the same value of entropy corresponds to two different values of the dispersion, which is clearly demonstrated on the graph (Fig. 1) constructed when $a=-1, b=1, \sigma^{2} \in[0,06 ; 0,7]$.

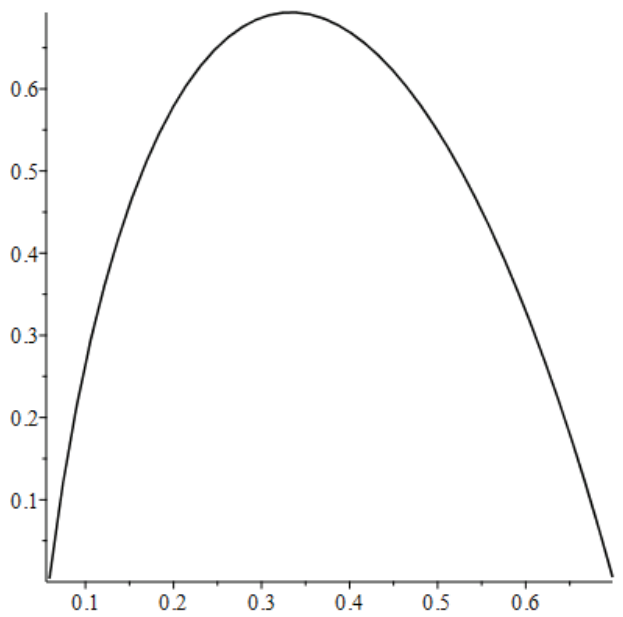

Fig. 1. Dependence of entropy on the value of dispersion

Is it true that this law provides the minimum and maximum dispersion $\mathrm{T}$ a given entropy. To this end, two inverse variational problems were being solved: the determination of the types of distribution densities ensuring the minimum and maximum dispersion of a random variable concentrated on a finite interval at a given entropy.

Both problems were solved for the case of a centered random variable.

Problem 1 which makes it possible to determine the form of the distribution density that ensures the minimum of the dispersion $D(x)$ for a given differential entropy $H_{\partial}$, is formulated as follows.

To determine the density $p(x)$ that delivers an extremum to the functional

$$
\min _{p(x)} \rightarrow\left\{D(x)=-\int_{a}^{b} x^{2} p(x) \cdot d x\right\},
$$

under the restrictions:

$$
\begin{gathered}
-\int_{a}^{b} p(x) \cdot \ln p(x) d x=H_{\partial}, \\
\int_{a}^{b} p(x) d x=1 .
\end{gathered}
$$

Thus, in this case there is a variational problem for a conditional extremum with isoperimetric constraints.

The Euler equation for the functional (3) and constraints (4), (5) takes the form:

$$
F_{p}^{* \prime}-\frac{d}{d t} F_{p^{\prime}}^{* \prime}=0
$$


Where $F_{p}^{* \prime}, F_{p^{\prime}}^{*^{\prime}}$ - are the derivatives of the Lagrange function with respect to $p(x)$ and with respect to $p^{\prime}(x)$. In this expression:

$$
F^{*}=F_{0}+\sum_{j=1}^{n} \lambda_{i} F_{i} ; \lambda_{i} \text { - are the constants; } n=2 \text {, and }
$$
the functions $F_{0}, F_{1}, F_{2}$ have the form: $F_{0}=-x^{2} p(x)$; $F_{1}=-p(x) \cdot \ln p(x) ; F_{2}=p(x)$.

Given that $\frac{d}{d t} F_{p^{\prime}}^{* \prime}=0$, we have:

$$
F_{p}^{* \prime}=\left[F_{0}+\left(\sum_{j=1}^{n} \lambda_{i} F_{i}\right)\right]^{\prime} p=0
$$

Substituting this expression into the Euler equation and taking into account the expressions for $F_{0}, F_{1}, F_{2}$ we will finally obtain:

$$
-x^{2}-\lambda_{1}[\ln p(x)+1]+\lambda_{2}=0 .
$$

This expression determines the form of the distribution density, which ensures the minimum of the dispersion $D(x)$ :

$$
p(x)=e^{-\frac{x^{2}}{\lambda_{1}}} e^{\frac{\lambda_{2}-\lambda_{1}}{\lambda_{1}}} .
$$

Substituting (6) into the constraints (4), (5) and numerically solving the obtained system of equations, we find the values of the coefficients $\lambda_{1}, \lambda_{2}$.

Having changed the variables and taking into account that

$$
\lambda_{1}=2 \sigma^{2} ; e^{\frac{\lambda_{2}-\lambda_{1}}{\lambda_{1}}}=M \text {, and } \lambda_{1}>0,
$$

we obtain expressions for the density and distribution function in the following form:

$$
\begin{gathered}
p(x)=M e^{-\frac{x^{2}}{2 \sigma^{2}}} ; \\
F(x)=M \int_{a}^{x} e^{-\frac{x^{2}}{2 \sigma^{2}}} d x .
\end{gathered}
$$

Then, in accordance with the Euler-Poisson integral and taking into account the truncation of the normal law, we have:

$$
M=\frac{C}{\sigma \sqrt{2 \pi}},
$$

where

$$
C=\frac{\sigma \sqrt{2 \pi}}{\int_{a}^{b} e^{-\frac{x^{2}}{2 \sigma^{2}}} d x} .
$$

Substituting (8) into (7), we obtain:

$$
p(x)=\frac{C}{\sigma \sqrt{2 \pi}} e^{-\frac{x^{2}}{2 \sigma^{2}}}, F(x)=\frac{C}{\sigma \sqrt{2 \pi}} \int_{a}^{x} e^{-\frac{x^{2}}{2 \sigma^{2}}} d x .
$$

Thus, the resulting law is abridged normal.

Consequently, the minimal dispersion at a given entropy, as well as the maximum entropy at a given dispersion, is provided by an abridged normal law.

Thus, it is proved that the law providing the given entropy and having a minimum dispersion, provided that $D(x)<\frac{(b-a)^{2}}{12}$ is an abridged normal law. Here $D(x)$ is the dispersion of the obtained law.

Let us define the functional relationship between dispersion and entropy. We substitute expression (6) into the constraints (4), (5):

$$
\begin{gathered}
-\int_{-\beta}^{\beta} e^{\frac{-x^{2}}{\lambda_{1}}} e^{\frac{\lambda_{2}-\lambda_{1}}{\lambda_{1}}} \cdot \ln e^{\frac{-x^{2}}{\lambda_{1}}} e^{\frac{\lambda_{2}-\lambda_{1}}{\lambda_{1}}} d x=H_{\partial}, \\
\int_{-\beta}^{\beta} e^{\frac{-x^{2}}{\lambda_{1}}} e^{\frac{\lambda_{2}-\lambda_{1}}{\lambda_{1}}} d x=1 .
\end{gathered}
$$

We submit expression (9) in the form:

$$
-\int_{-\beta}^{\beta} e^{-\frac{x^{2}}{\lambda_{1}}} e^{\frac{\lambda_{2}-\lambda_{1}}{\lambda_{1}}}\left(\ln e^{\frac{-x^{2}}{\lambda_{1}}}+\ln e^{\frac{\lambda_{2}-\lambda_{1}}{\lambda_{1}}}\right) d x=H_{\partial}
$$

Having transformed this expression, taking into account (10), we obtain

$$
D(x)=\left(H_{\partial}-1\right) \lambda_{1}+\lambda_{2} .
$$

Expression (11) uniquely characterizes the relationship between the minimal dispersion $D(x)$ and the given differential entropy $H_{\partial}$ under the condition $D(x)<\frac{(b-a)^{2}}{12}$.

Problem 2, which makes it possible to determine the form of the distribution density that provides the maximum of the dispersion $D(x)$ for a given differential entropy $H_{\partial}$, differs from the previous one only in the form of the functional:

$$
\max _{p(x)} \rightarrow\left\{D(x)=\int_{a}^{b} x^{2} p(x) \cdot d x\right\}
$$

Similarly solving this problem we get: 


$$
p(x)=e^{\frac{x^{2}}{\lambda_{1}}} e^{\frac{\lambda_{2}-\lambda_{1}}{\lambda_{1}}} .
$$

Provided that $\lambda_{1}>0$ the law obtained corresponds to the one bimodal.

The results of the simulation are shown in Fig. 2.
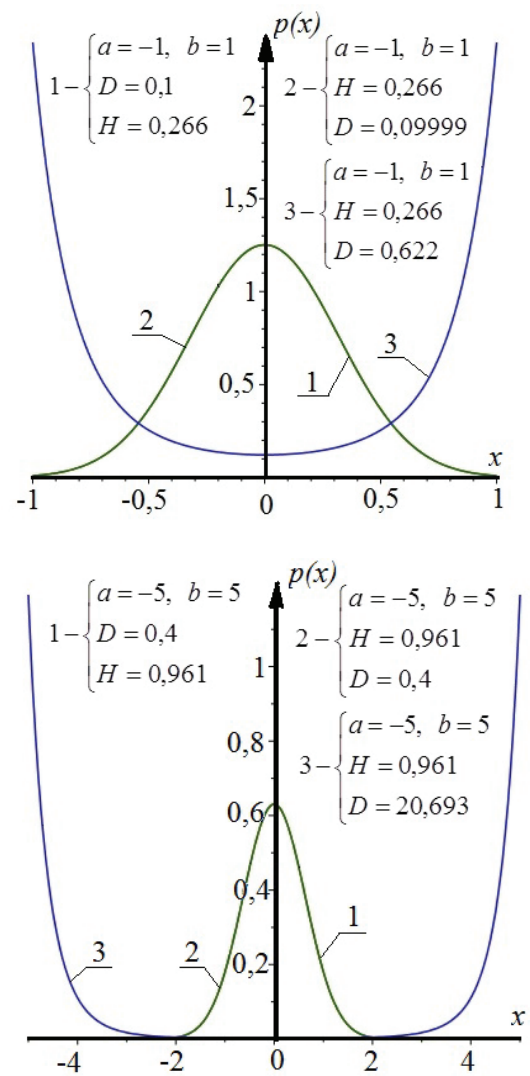

1 - abridged normal

2 - law ensuring minimum dispersion

3 - law ensuring maximum of dispersion

Fig. 2. Results of modeling

As for the problem of determining the dispersion minimum, we define a functional connection between the maximum dispersion $D(x)$ and the given differential entropy $H_{\partial}$.

Let us substitute the expression for the distribution density (12) into the constraints (4), (5):

$$
\begin{gathered}
-\int_{-\beta}^{\beta} e^{\frac{x^{2}}{\lambda_{1}}} e^{\frac{\lambda_{2}-\lambda_{1}}{\lambda_{1}}} \cdot \ln e^{\frac{x^{2}}{\lambda_{1}}} e^{\frac{\lambda_{2}-\lambda_{1}}{\lambda_{1}}} d x=H_{\partial}, \\
\int_{-\beta}^{\beta} e^{\frac{x^{2}}{\lambda_{1}}} e^{\frac{\lambda_{2}-\lambda_{1}}{\lambda_{1}}} d x=1 .
\end{gathered}
$$

We represent expression (13) in the form:

$$
-\int_{-\beta}^{\beta} e^{\frac{x^{2}}{\lambda_{1}}} e^{\frac{\lambda_{2}-\lambda_{1}}{\lambda_{1}}}\left(\ln e^{\frac{x^{2}}{\lambda_{1}}}+\ln e^{\frac{\lambda_{2}-\lambda_{1}}{\lambda_{1}}}\right) d x=H_{\partial}
$$

Converting this expression to (14)
We obtain a functional relationship between the maximum dispersion and the given entropy $H_{\partial}$.

$$
-D(x)=\left(1-H_{\partial}\right) \lambda_{1}-\lambda_{2} .
$$

The expression (15) uniquely characterizes the relationship between the maximum dispersion $D(x)$ and the given differential entropy $H_{\partial}$ under the condition $D(x)>\frac{(b-a)^{2}}{12}$.

The results obtained can be formulated as a theorem.

Theorem on the relation of a dispersion to the differential entropy of a continuous centered random variable concentrated on a finite interval. If the continuous centered random variable is concentrated on a finite interval bounded by the limiting values $a, b$, then, under the condition $D(x)<\frac{(b-a)^{2}}{12}$ the minimum dispersion $D(x)$ and the differential entropy $H_{\partial}$ of the indicated random variable are related by a relation $D(x)=\left(H_{\partial}-1\right) \lambda_{1}+\lambda_{2}$, where the constants $\lambda_{1}$ and $\lambda_{2}$ are defined as solutions of the equations system

$$
\begin{gathered}
-\int_{a}^{b} e^{-\frac{x^{2}}{\lambda_{1}}} e^{\frac{\lambda_{2}-\lambda_{1}}{\lambda_{1}}} \cdot \ln e^{-\frac{x^{2}}{\lambda_{1}}} e^{\frac{\lambda_{2}-\lambda_{1}}{\lambda_{1}}} d x=H_{\partial} ; \\
\int_{a}^{b} e^{-\frac{x^{2}}{\lambda_{1}}} e^{\frac{\lambda_{2}-\lambda_{1}}{\lambda_{1}}} d x=1
\end{gathered}
$$

and when the condition $D(x)>\frac{(b-a)^{2}}{12}$ is satisfied, the differential entropy $H_{\partial}$ and the maximum dispersion $D(x)$ of the indicated random variable are related by the correlation $-D(x)=\left(H_{\partial}-1\right) \lambda_{1}+\lambda_{2}$, where the constants $\lambda_{1}$ and $\lambda_{2}$ are defined as the solutions of the equations systems:

$$
\begin{gathered}
-\int_{a}^{b} e^{\frac{x^{2}}{\lambda_{1}}} e^{\frac{\lambda_{2}-\lambda_{1}}{\lambda_{1}}} \cdot \ln e^{\frac{x^{2}}{\lambda_{1}}} e^{\frac{\lambda_{2}-\lambda_{1}}{\lambda_{1}}} d x=H_{\partial} \\
\int_{a}^{b} e^{-\frac{x^{2}}{\lambda_{1}}} e^{\frac{\lambda_{2}-\lambda_{1}}{\lambda_{1}}} d x=1 .
\end{gathered}
$$

Consequence. The extremal values of the dispersion for a given differential entropy of a continuous centered random variable concentrated on a finite interval bounded by the limiting values $a, b$, are uniquely provided by the compositional law of the form:

$$
p(x)=e^{\mu_{1}-1} \cdot e^{\mu_{2} \cdot x^{2}}, \quad a \leq x \leq b,
$$

where the constants $\mu_{1}$ and $\mu_{2}$ are defined as solutions of the equations system 


$$
\begin{gathered}
\int_{a}^{b} e^{\mu_{1}-1} \cdot e^{\mu_{2} \cdot x^{2}} d x=1 ; \\
\int_{a}^{b} x^{2} \cdot e^{\mu_{1}-1} \cdot e^{\mu_{2} \cdot x^{2}} d x=D_{3},
\end{gathered}
$$

where $D_{3}$ is the specified dispersion value.

On the basis of the studies carried out it can be concluded that the abridged normal distribution law ensures a maximum of entropy for a given dispersion and at the same time a minimum of dispersion at a given entropy, and the bimodal law provides the maximum of entropy at the maximum of the dispersion.

Let us note once again that the concept of entropy which is a measure of uncertainty in the information theory, in this case, differs with the concept of uncertainty of the measurement accuracy used as a certainty criterion and given in $[15,16]$, and the correlations (12) and (17) relate these two concepts with unique functional dependencies.

Another important conclusion that can be drawn from the studies is that the distribution density obtained from the maximum of the entropy of a random variable concentrated on a finite interval for a given dispersion uniquely corresponds to the distribution densities of the laws that ensure the minimum and maximum of the dispersion for the same entropy.

As further research there will be the analysis of the applicability of the obtained distribution law in various areas of mechanical assembly production in the machine-tool industry.

\section{References}

1. L.N. Gavrilov, N.A. Borodachev, R.M. Abrashitov. Accuracy of production in mechanical engineering and instrument making (Mechanical Engineering, Moscow, 1973)

2. N.G. Bruevich., E.A. Pravotorova, V.I. Sergeev. Fundamentals of the theory of the accuracy of mechanisms: (Nauka, Moscow, 1988)

3. V.P. Bulatova (red.), I.G. Friedlander (red.), Fundamental problems of the theory of accuracy. (Science, St. Petersburg, 2001)

4. RMG 64-2003 "GSI. Ensuring efficiency in the management of technological processes. Methods and ways to improve the accuracy of measurements »

5. V.Ya. Kopp, A.A. Skidan, A.I. Balakin, O.V. Filipovich. Proceedings of the Odessa Polytechnic University. Analysis of differential entropy in technical measurements in machine-tool construction, 1(27). PP. 214 - 219 (2007)

6. V.Ya. Kopp, A.A. Skidan, A.I. Balakin, O.V. Filipovich. Zb. Sciences. The praise of SNYAETA. Optimum estimation of the number of measurements of instrument systems, 1(21). PP.231 -238 (2007)

7. V.I. Dmitriev. Applied information theory (Higher education. Shk. Moscow, 1989).

8. P.V. Novitsky. Fundamentals of the information theory of measuring devices. (Energia, Leningrad, 1968).

9. P.V. Novitsky, I.A. Zograf Estimation of errors in measurement results (Energoatomizdat, Leningrad 1991).

10. E.T. Jane. Proceedings of the Institute of Electrical and Electronics Engineers. On the logical justification of the maximum entropy methods, 9(70). PP. 33 - 51 (1982).

11. V.Ya. Kopp, A.A. Skidan, A.P. Vasyutenko. Bulletin of SevNTU. Process Automation and Control. Optimum estimation of the number of measurements of instrument systems, (36). PP. $75-$ 80 (2002).

12. GOST 8.207-76 State system for ensuring the uniformity of measurements. Direct measurements with multiple observations. Methods for processing the results of observations. Basic provisions.

13. GOST R ISO 5725-2002. Accuracy (correctness and precision) of measurement methods and results. Part 2.

14. RMG 43-2001 State system for ensuring the uniformity of measurements. Application of the "Guide to the expression of measurement uncertainty".

15. T. Barashkova, R. Laaneots. Uncertainty of the result of measurements (Tallinn Technical University, Tallinn, 2003).

16. I.P. Zakharov, V.D. Kukush. Theory of uncertainty in measurements (Konsum, Kharkov, 2002). 